\title{
The Influence of Parent-Child Intimacy, Rejection Sensitivity, and Empathy on Dating Relationship Control in Women College Students
}

\author{
Sunghee Lee ${ }^{1}$ and Seolhee Son ${ }^{2}$ \\ ${ }^{1}$ Professor, College of Nursing, The Research Institute of Nursing, Kyungpook \\ National University, 680, Gukchaebosang-ro, Jung-gu, Daegu 41944, Korea \\ ${ }^{2}$ Graduate Student, Graduate School of Nursing, Kyungpook National University, 680, \\ Gukchaebosang-ro, Jung-gu, Daegu 41944, Korea \\ ${ }^{1}$ leesh@knu.ac.kr, ${ }^{2}$ seolheeson@empas.com
}

\begin{abstract}
The purpose of this study was to identify the influence of parent-child intimacy, rejection sensitivity, and empathy on dating relationship control of female students. The data were collected from June to July 2016 through structured questionnaires from 116 female college students recruited through liberal arts classes in four universities located in D metropolitan city. The collected data were analyzed using the IBM SPSS Statistics 21 program. Stepwise multiple regression was carried out to identify the influence of parent-child intimacy, rejection sensitivity, and empathy on dating relationship control in female college students. It was found that rejection sensitivity ( $\beta=-.210, p=.003)$ and emotional empathy $(\beta=.261$, $p=.008)$ were some of the factors influencing dating relational control among female college students. These variables explained $10.0 \%$ of the variance in dating relational control $(F=5.244, p=.002)$. Female college students had a greater control over dating relationship when they had high emotional empathy and low rejection sensitivity.
\end{abstract}

Keywords: Control, Dating, Empathy, Relationship, Rejection

\section{Introduction}

\subsection{Study rationale}

Relationship control is a concept based on the theory of sexual power that claims men hold more control than women in various decision making processes since gender inequality is still dominant in society [1]. Dating relationship control, in particular, refers to a power that allows people to express their thoughts and feelings, to do what they want in their own way, and to have a greater voice in decision making [2].

According to the sexual power theory, if a woman has less control over dating relationship, it is difficult for her to get an upper hand in decision-making. Thus, women with low dating relationship control are more likely to fall victim to intimate relationship violence including dating abuse, and have difficulty in asserting their thoughts and needs that go against those of men in an intimate relationship, resulting in a variety of negative consequences [1].

According to Pulerwitz et al. [1], relationship power plays a key role in encouraging

Article history:

Received (August 02, 2016), Review Result (September 27, 2016), Accepted (October 29, 2016) 
women to engage in positive health behavior, such as protecting substance-addicted women from risky sexual practices. Also, women with greater relationship power were found to be 6.17 time more likely to use a condom for three months or longer when having sex with a partner [2]. By contrast, women with low relationship control were at a higher risk of being infected with HIV than those with moderate or high relationship control [3]. Low relationship control affects women not only in dating relationship, but also with regard to domestic violence since women with low relationship power have a higher chance of being exposed to abuse in intimate relationship [4]. Furthermore, a research proved that female college students who have high control of dating relationship were more likely to refuse to have unwanted sex [5]. In this regard, there is a need for an intervention that could increase female college students' dating relationship control, thereby protecting them from having risky and unwanted sex. However, a research that influences dating relationship control should be preceded a base of such an intervention development. As such, this study was designed to investigate factors affecting female college students' dating relationship control by examining their parent-child intimacy, rejection sensitivity, and empathy. We expect that the study findings would serve as the baseline data for development of an intervention program that could improve female college students' control of dating relationship.

\section{Methods}

\subsection{Participants}

This study was conducted on 116 female students who were attending four universities in city D. We calculated an appropriate sampling size through $\mathrm{G}^{*}$ power 3.1 after setting significance level at .05 , power of test at .90 , effect size at .15, and the number of predictors at 5. As a result, we found that the minimum number of samples required for the study was 116 . Considering the possibility of students not responding or not having a prior sexual relationship experience, we decided to collect data from 250 students. When we selected the study subjects, we checked whether they had ever been in a sexual relationship and if they were willing to participate in the study.

Among 232 copies of the questionnaire collected from participating students, we removed 82 since they were from those who reported they hadn't had a sexual relationship before. We also removed responses from 34 students who didn't give a valid answer regarding parent-child intimacy. As a result, we ultimately utilized 116 copies of the survey for statistical analysis.

\subsection{Measurement tools}

\subsubsection{Parent-child intimacy}

Parent-child intimacy was measured by using the Parent-Child Affective Bonding Scale developed by Lee JJ [6]. It was developed on the ground that children's thoughtfulness was affected by how close psychological and emotional relationships they had with their parents and whether they received strong support from parents. This tool consisted of two sub-categories such as reliability and devotion, and comprised of 40 questions: 20 on father-child intimacy and 20 on mother-child intimacy. Respondents were asked to give 1 (not at all) to 5 (very much) points for each question on a 5-point Likert scale, meaning they could give 20-100 points in each category. Also, getting higher points indicated having more intimate relationship with father or mother. While Lee JJ yielded Cronbach's $\alpha$ of .90-92 in his study, 
Cronbach's alphas of parent-child intimacy, father-child intimacy, and mother-child intimacy were $.97, .95$, and .95 , respectively, in this study.

\subsubsection{Rejection sensitivity}

To examine rejection sensitivity, we employed a Korean version of the Rejection Sensitivity Questionnaire (RSQ) invented by Downey and Feldman [7], and translated and modified by Lee BD [8]. This tool presented 18 different situations of daily life pertaining to early stage of adulthood that the participants requested the important people in their lives, such as parents, friends, professors or lovers, to accept in order to assess the rejection sensitivity under each situation. The respondents were supposed to measure whether they were concerned or anxious about their request being rejected (expectation of rejection) and whether they believed their demand would be accepted or not, with giving 1 (Never) to 6 (Very much) points on a six-point Likert scale.

To measure rejection sensitivity of each situation, we counted backwards the points that the subject gave for expectation of acceptance in each situation, multiplied the results by the points she marked for anxiety and expectation about being rejected in the same situation. Next, we added the multiplied points for all 18 situations and divided the aggregate value by 18. The total score could range from 1 to 36 and scoring higher points meant higher sensitivity toward rejection. The tool's Cronbach's $\alpha$ in Lee DB [8]'s study was .90 where as it recorded Cronbach's $\alpha$ of .91 in this study.

\subsubsection{Empathy}

We examined the subjects' empathy by using Davis [9]'s Interpersonal Reactivity Index and Emotional Empathy Scale, which was invented by Mehrabian and Esptein [10] and modified by Song GH and Cho GP [11] to measure cognitive and emotional empathy. This method comprised a total of 33 questions: 6 for cognitive empathy and 27 for emotional empathy. Based on a five-point Likert scale, the subjects were asked to give 1 (Never) to 5 (Absolutely) points for each of the questions and 12 of the negative questions were reversely coded. In other words, respondents could score 6-30 points for cognitive empathy and 27-135 for emotional empathy, and getting higher points meant having more empathic capacity. While the tool's Cronbach's $\alpha$ was .77 in Song GH and Cho GP [11]'s research, this study found the tool's Cronbach's $\alpha$ standing at .84. More specifically, the tool's Cronbach's $\alpha$ was .72 for the cognitive empathy test and .85 for the emotional empathy test in the study.

\subsubsection{Dating relationship control}

Dating relationship control was measured by using relationship power scale, a sub-category of the Sexual Relationship Power Scale, which was developed by Pulerwitz et al. and translated and modified by Yoo OS [5]. Respondents marked points on a total 12 questions based on a four-point Likert scale. Therefore, they gave 1 for "Never," 2 for "No," 3 for "Yes," and 4 for "Absolutely." The total score could range from 12 to 48 and scoring higher points meant getting more control of dating relationship. This tool marked Cronbach's $\alpha$ of .71 in the current study while recording Cronbach's $\alpha$ of .84 in Yoo OS's study [5].

\subsection{Data collection}

Data were collected from June to July of 2016. Researchers received an approval for data 
collection from a professor who was providing eight summer sessions at four colleges located in city D. During these permitted classes, we briefed the students on the purpose of the study and details of the questionnaire. We, then, informed them that the survey would be performed anonymously to guarantee confidentiality and if they didn't want to respond, they could always withdraw from the survey. Finally, we explained that the collected data would be used for the present study only, obtained written consents from the students willing to participate, and then began collecting data. It took about 15 minutes for the subjects to completely fill in the survey form.

\subsection{Data analysis}

We analyzed the collected data by utilizing IBM SPSS Statistics 21.0. The subjects' general characteristics were measured by frequency and percentage; in addition, parent-child intimacy, rejection sensitivity, empathy, and dating relationship were analyzed by the mean value and standard deviation. To examine differences in the subjects' dating relationship control depending on their general characteristics, we carried out t-test and ANOVA. We also used Pearson's correlation coefficient to investigate correlations among parent-child intimacy, rejection sensitivity, empathy, and dating relationship control of the subjects. Finally, we performed a stepwise multiple regression analysis to look into factors affecting the subjects' control over dating relationship.

\section{Results}

\subsection{General characteristics of the subjects}

$27.6 \%$ of the study subjects reported that they followed a religion and, by grade, $40.5 \%$ or the biggest proportion of them were third-year students. The average GPA that the subjects scored in the previous term was $3.56( \pm 0.45)$. In all, $31.6 \%$ had a higher GPA than the average, while $68.4 \%$ got lower GPAs than that. In terms of dating experience with the opposite sex, $12.9 \%$ said $1-2$ times and $87.1 \%$ responded three times or more.

\subsection{Parent-child intimacy, rejection sensitivity, empathy and dating relationship control}

The subjects scored the average of $164.87( \pm 24.85)$ in parent-child intimacy. More specifically, they recorded $81.44( \pm 13.37)$ and $83.43( \pm 12.27)$ on an average in father-child intimacy and mother-child intimacy, respectively. In terms of rejection sensitivity, the subjects marked the mean points of $7.99( \pm 2.98)$. In addition, they received $120.19( \pm 12.32)$ for empathy: $21.94( \pm 3.29)$ for cognitive empathy and $98.25( \pm 10.10)$ for emotional empathy. Finally, they averaged at $36.67( \pm 4.25)$ for dating relationship control.

\subsection{Relationship among parent-child intimacy, rejection sensitivity, empathy and dating relationship control}

We looked into the correlation among subjects' parent-child intimacy, rejection sensitivity, and dating relationship control and found that rejection sensitivity had a negative correlation ( $\mathrm{r}=-.254, p=.006)$ with dating relationship control while empathy had a positive correlation $(\mathrm{r}=.184, p=.049)$ with the control. 


\subsection{Factors affecting subjects' control of dating relationship}

The regression model marked the Durbin-Watson value of 1.791 , indicating there was no self-correlation, and its tolerance was .833-.850, which was higher than 0.1. Moreover, variance inflation factors stood at 1.162-1.201, less than 10, meaning all variables had no issue of multicollinearity.

The stepwise multiple regression analysis verified the rejection sensitivity $(\beta=-.210, p=.003)$ and empathy $(\beta=.261, p=.008)$ as factors that influence female college students' control of dating relationship, and the two variables turned out to explain $10.0 \%$ of the dating relationship control $(\mathrm{F}=5.244, p=.002)$. Empathy, in particular, had greater effect on the students' control. From these results, this study confirmed that female college students, who are less sensitive to rejection but feel more empathy, have higher control over dating relationship [Table 1].

Table 1. Factors influencing dating relationship control among subjects $(\mathrm{N}=116)$

\begin{tabular}{|c|c|c|c|c|c|c|c|}
\hline Variables & $\mathrm{B}$ & $\mathrm{B}$ & $\mathrm{t}$ & $p$ & $\mathrm{R}^{2}$ & Adj. $\mathrm{R}^{2}$ & $\mathrm{~F}(p)$ \\
\hline (Constant) & 33.144 & - & 7.837 & $<.001$ & .123 & .100 & $\begin{array}{c}5.244 \\
(.002)\end{array}$ \\
\hline Rejection sensitivity & -.017 & -.210 & -2.199 & .030 & - & - & - \\
\hline Emotional empathy & .123 & .261 & 2.704 & .008 & - & - & - \\
\hline
\end{tabular}

\section{Conclusion and suggestions}

This present study was designed to investigate the factors that affect female college students' control over dating relationship, thereby providing the baseline data for development of a nursing intervention program that could help female college students gain greater control over dating relationship. The study found that rejection sensitivity and empathy influenced female college students' control of dating relationship.

A tool that would inspect these students' dating relationship control as well as an intervention program that could help them obtain more control could be developed based on the factors this study identified. As such, we'd like to make the following suggestions.

First, a replication study needs to be conducted covering nation-wide samples.

Next, it is required to develop and apply a tool that can investigate female college students' control over dating relationship and risk factors as well as a nursing intervention program to improve the control.

\section{References}

[1] J. Pulerwitz, H. Amaro, W. De Jong, S. L. Gortmaker, and R. Rudd, "Relationship power, condom use and HIV risk among women in the USA," AIDS Care, vol.14, no.6, (2002) DOI:10.1080/0954012021000031868

[2] J. Pulerwitz, S. L. Gortmaker, and W. De Jong, "Measuring sexual relationship power in HIV/STD research," Sex Roles, vol.42, pp.7-8, (2000)

[3] R. K. Jewkes, K. Dunkle, M. Ndun, and N. Shai, "Intimate partner violence, relationship power inequity, and incidence of HIV infection in young women in South Africa: a cohort study,” The Lancet, vol.376, pp.9734, (2010) DOI:10.1016/S0140-6736(10)60548-X

[4] J. C. Campbell, P. Miller, M. M. Cardwell, and R. A. Belknap, "Relationship status of battered women over time," Journal of Family Violence, vol.9, no.2, (1994) DOI:10.1007/BF01531957

[5] O. S. Yoo, "An Explanatory model of unwanted sexual compliance with dating partners among college Students,” Ph.D. dissertation, Seoul Women's University, Seoul, (2004) 
The Influence of Parent-Child Intimacy, Rejection Sensitivity, and Empathy on Dating Relationship Control in Women College Students

[6] J. J. Lee, "A study on the construction and validation of parent-adolescent affective bonding (Bu-Ja-Uy-Chin-Sung-Cheong) scale,” Ph.D. dissertation, Chung-Ang University, Seoul, (2002)

[7] G. Downey and S. I. Feldman, "Implications of rejection sensitivity for intimate relationships," Journal of Personaliy and Social Psychology, vol.70, no.6, (1996) DOI:10.1037/0022-3514.70.6.1327

[8] B. D. Lee, "The relationship between adult attachment and satisfaction of dating relationship-focusing on the mediating effect of rejection sensitivity and attribution style," M.S. thesis, Korea University, Seoul, (2000)

[9] M. H. Davis, “A multidimensional approach to individual differences in empathy,” APA, (1980)

[10] A. Mehrabian and N. Epstein, "A measure of emotional empathy," Journal of Personality, vol.40, no.4, (1972) DOI:10.1111/j.1467-6494.1972.tb00078.x

[11] G. H. Song and G. P. Cho, "Analysis of the structural relationship among moral self, self-control, empathy and moral behavior of university students," Korean Association for Learner-centered Curriculum and Instruction, vol.13, pp.23-44, (2013) 